\title{
Gapless Spin-Liquid Phase in an Extended Spin 1/2 Triangular Heisenberg Model
}

\author{
Ryui Kaneko ${ }^{1}$, Satoshi Morita ${ }^{2}$, and Masatoshi Imada ${ }^{3}$ \\ ${ }^{1}$ Institut für Theoretische Physik, Goethe-Universität Frankfurt, Max-von-Laue-Straße 1, 60438 Frankfurt \\ am Main, Germany \\ ${ }^{2}$ Institute for Solid State Physics, University of Tokyo, Kashiwa, Chiba 277-8581, Japan \\ ${ }^{3}$ Department of Applied Physics, University of Tokyo, Bunkyo, Tokyo 113-8656, Japan
}

\begin{abstract}
We numerically study the Heisenberg models on triangular lattices by extending it from the simplest equilateral lattice with only the nearest-neighbor exchange interaction. We show that, by including an additional weak next-nearest-neighbor interaction, a quantum spin-liquid phase is stabilized against the antiferromagnetic order. The spin gap (triplet excitation gap) and spin correlation at long distances decay algebraically with increasing system size at the critical point between the antiferromagnetic phase and the spin-liquid phase. This algebraic behavior continues in the spin-liquid phase as well, indicating the presence of an unconventional critical (algebraic spin-liquid) phase characterized by the dynamical and anomalous critical exponents $z+\eta \sim 1$. Unusually small triplet and singlet excitation energies found in extended points of the Brillouin zone impose constraints on this algebraic spin liquid.
\end{abstract}

The spin liquid was first proposed by Anderson as a resonating valence bond (RVB) ground state of the spin $1 / 2$ antiferromagnetic Heisenberg model on the triangular lattice. ${ }^{1)}$ Although it is now widely believed that the true ground state of the model has a long-ranged magnetic order, ${ }^{2-4)}$ two-dimensional systems based on some modification of the triangular Heisenberg model provide us with realistic possibilities of reaching the spin liquid. This is because some organic conductors are basically described by the triangular structure with some additional elements, and they indeed do not show clear indications of symmetry breaking. ${ }^{5,6)}$ If one can understand how and in which direction the spin-liquid state becomes more stabilized and what kind of spin liquids is expected even at the level of model studies, it will greatly help as a clue for stabilizing spin liquids.

In this letter, we study the Heisenberg model on the triangular lattice shown in Fig. 1(a) with a weak nextnearest-neighbor interaction $J_{2}$ illustrated in the inset of Fig. 1(b). The Hamiltonian has the form

$$
H=J_{1} \sum_{\langle i, j\rangle} \boldsymbol{S}_{i} \cdot \boldsymbol{S}_{j}+J_{2} \sum_{\langle\langle i, j\rangle\rangle} \boldsymbol{S}_{i} \cdot \boldsymbol{S}_{j},
$$

where $\boldsymbol{S}_{i},\langle i, j\rangle$, and $\langle\langle i, j\rangle\rangle$ denote the quantum $S=1 / 2$ spin at site $i$, nearest-neighbor sites, and next-nearestneighbor sites, respectively.

Previous studies ${ }^{7-16)}$ have suggested that the above model contains rich phases, as shown in Fig. 1(c). For the classical Heisenberg model, the $120^{\circ}$ Néel state illustrated in Fig. 1(b) is stable for $J_{2} / J_{1}<1 / 8$, while for $J_{2} / J_{1}>1 / 8$ the ground states become degenerate for any four-sublattice states that satisfy $\boldsymbol{S}_{1}+\boldsymbol{S}_{2}+\boldsymbol{S}_{3}+$ $\boldsymbol{S}_{4}=0$, where $\boldsymbol{S}_{i}$ denotes the spin at sublattice site $i$. When $J_{2} / J_{1}>1$, an incommensurate spin structure appears. By considering quantum fluctuations perturbatively, the stripe-type antiferromagnetic state illustrated in Fig. $1(\mathrm{~b})^{8-14)}$ was proposed for $1 / 8<J_{2} / J_{1}<1$.
Then, the spin-liquid state could emerge at approximately $J_{2} / J_{1}=1 / 8$, sandwiched by the $120^{\circ}$ Néel and stripe states. $\left.{ }^{8}, 10\right)$ Very recently, by using a variational Monte Carlo (VMC) method, Mishmash et al. claimed that the spin-liquid phase with the nodal $d$-wave symmetry is realized for $0.05 \lesssim J_{2} / J_{1} \lesssim 0.17 .{ }^{16)}$ However, they overestimated the spin-liquid phase, since their HuseElser-type wave functions for antiferromagnetic states are relatively inaccurate. Moreover, their wave function favors the $d$-wave-type spin liquid due to a limited number of variational parameters.

To reveal the nature of the spin-liquid ground states possibly realized in this model, we calculate the ground states and low-energy excitations up to $18 \times 18$ sites by a many-variable variational Monte Carlo (mVMC) method $^{17)}$ (see Supplemental Materials ${ }^{18)}$ for more details). We find a spin-liquid phase characterized by gapless excitations and the power-law decay of the spin correlation with the critical and dynamical exponents $\eta$ and $z$, respectively, satisfying $z+\eta=1$ in the parameter region $0.10(1) \leq J_{2} / J_{1} \leq 0.135(5)$, as shown in Fig. 1(b).

Three locally stable states are found as candidates of the ground state: the $120^{\circ}$ Néel, stripe, and spin-liquid states. As shown in Fig. 2, these states are characterized by the spin structure factor defined as

$$
S(\boldsymbol{q})=\frac{1}{N_{\mathrm{s}}} \sum_{i, j} e^{i \boldsymbol{q} \cdot\left(\boldsymbol{R}_{i}-\boldsymbol{R}_{j}\right)}\left\langle\boldsymbol{S}_{i} \cdot \boldsymbol{S}_{j}\right\rangle,
$$

where $\boldsymbol{R}_{i}$ denotes the position vector of site $i$. The existence of the long-ranged order is ensured when the maximum of $S(\boldsymbol{q})$ at $\boldsymbol{q}=\boldsymbol{Q}$ scales as $S(\boldsymbol{Q}) \propto N_{\mathrm{s}}$ for a large $N_{\mathrm{s}}$. The ground state is determined from the comparison of the energy, which is extrapolated to the thermodynamic limit, if more than one locally stable states are found.

For $J_{2} / J_{1}<0.1$, we find the $120^{\circ}$ Néel state as the ground state. In the Brillouin zone illustrated in 

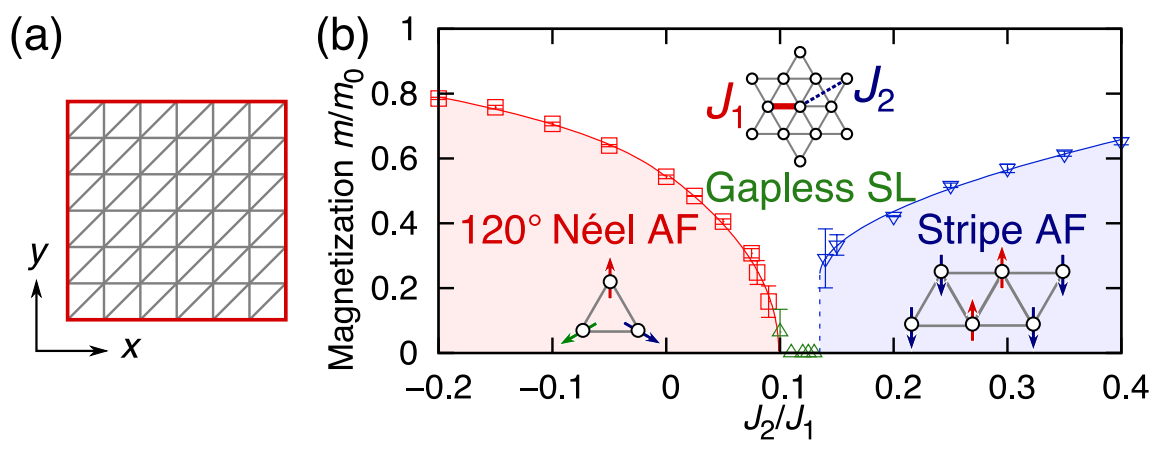

(d)

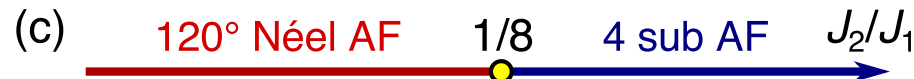

Fig. 1. (color online) (a) Deformed triangular lattice, which is topologically equivalent to isotropic triangular lattice. (b) Phase diagram of antiferromagnetic $J_{1}-J_{2}$ Heisenberg model on triangular lattice obtained in the present study. The red squares, green up-pointing triangular points, and blue down-pointing triangular points are order parameters (sublattice magnetizations) of the $120^{\circ}$ Néel states $\left(120^{\circ} \mathrm{Néel} \mathrm{AF}\right)$, spin-liquid (SL) states, and stripe states (stripe AF), respectively. The lines are guides for the eyes. The magnetization is estimated as $m / m_{0}=2 \sqrt{c \times \lim _{N_{\mathrm{s}} \rightarrow \infty} S(\boldsymbol{Q}) / N_{\mathrm{s}}}$, where $c$ denotes a correction factor, and $S(\boldsymbol{Q})$ is the peak value of the spin structure factor. For the $120^{\circ}$ Néel and SL states, $c=2$, which is compatible with the standard definition, ${ }^{2-4)}$ while for the stripe states, $c=1$. Here, $m_{0}(=1 / 2)$ is the saturated magnetization expected in the classical Néel order. (c) Phase diagram of classical model as reference. The transition from the $120^{\circ}$ Néel AF state to the four-sublattice antiferromagnetic state ( $4 \mathrm{sub} \mathrm{AF}$ ) occurs at $J_{2} / J_{1}=1 / 8$. The stripe $\mathrm{AF}$ state is one of the 4 sub-AF states, and it is selected under quantum fluctuations. (d) Brillouin zone of deformed triangular lattice. Equivalent momenta are represented by the same symbols.
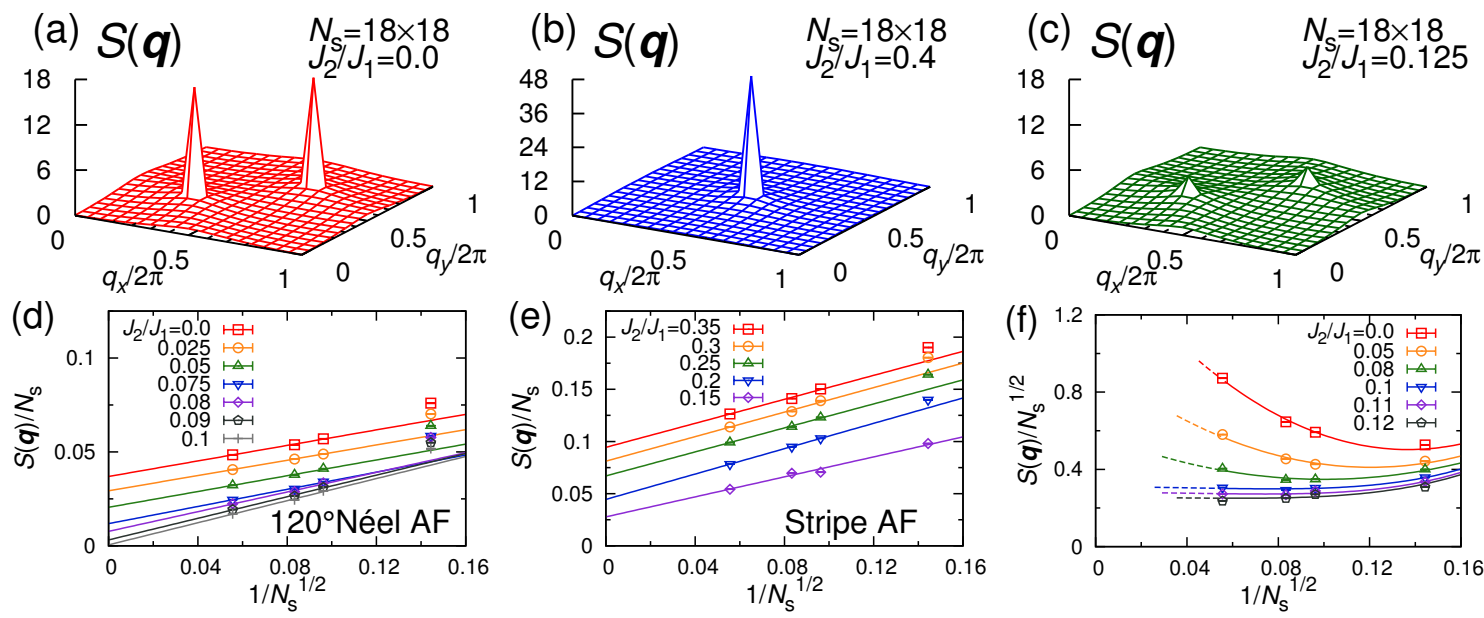

Fig. 2. (color online) (a-c) Spin structure factors $\left(N_{\mathrm{s}}=18 \times 18\right)$ for $120^{\circ}$ Néel state at $J_{2} / J_{1}=0(\mathrm{a})$, stripe state at $J_{2} / J_{1}=0.4(\mathrm{~b})$, and spin-liquid state with short-ranged magnetic correlation with the $120^{\circ}$ spin alignment at $J_{2} / J_{1}=0.125$ (c). (d-f) Size dependences of peak value of $S(\boldsymbol{Q}) / N_{\mathrm{s}}$ for $120^{\circ}$ Néel states (except $\left.J_{2} / J_{1}=0.1\right)(\mathrm{d})$, stripe states (e), and $S(\boldsymbol{Q}) / N_{\mathrm{s}}^{1 / 2}$ for $120^{\circ}$ Néel states and spin liquid states (f). Size dependence at $J_{2} / J_{1}=0.1$ is also shown in (d) for reference. We extrapolate the data using the relation $S(\boldsymbol{Q}) / N_{\mathrm{s}}=m^{2} / c+S_{1} / N_{\mathrm{s}}^{1 / 2}+S_{2} / N_{\mathrm{s}}$, where $m$ denotes the extrapolated magnetization, $c$ denotes a correction factor, which is the same as in Fig. 1(a), while $S_{1}$ and $S_{2}$ are constants. For antiferromagnetic states, we use the data for $N_{\mathrm{s}}>100$ to reduce the finite-size effect, and fit them by the relation with $S_{2}=0$ to estimate the statistical error bars. On the other hand, to precisely determine the phase boundary for spin-liquid states, we also examine the size dependence of $S(\boldsymbol{Q})$. We find that $S(\boldsymbol{Q})$ scales as $S(\boldsymbol{Q}) \propto N_{\mathrm{s}}^{1 / 2}$ for $J_{2} / J_{1} \geq 0.1$.

Fig. 1(d), Bragg peaks appear at $\boldsymbol{q}= \pm(2 \pi / 3,2 \pi / 3)$ corresponding to the $120^{\circ}$ spin structure, as shown in Fig. 2(a). From the size extrapolation of $S(\boldsymbol{Q}) / N_{\mathrm{s}}$ in Fig. 2(d), we obtain the magnetization of the $120^{\circ}$ Néel state at $J_{2} / J_{1}=0$ as $m / m_{0}=0.543(6)$, where $m_{0}=1 / 2$ is the saturated magnetization in spin $1 / 2$ systems. This value is nearly the same as the best variational result $\left(m / m_{0}=0.53\right)$ in the literature. ${ }^{19)}$ For $J_{2} / J_{1}>0.135$, we find the stripe ordered state as the ground state. As shown in Fig. 2(b), a Bragg peak appears at $\boldsymbol{q}=(\pi, \pi)$, corresponding to the ferromagnetic spin alignment along the $x=y$ direction and the antiferromagnetic spin alignment along the $x$ - and $y$-axes on the lattice illustrated in Fig. 1(a). The state has a long-ranged order, as shown in Fig. 2(e).

Sandwiched by the $120^{\circ}$ Néel and stripe states, the spin liquid is stabilized. Diffusive peaks appear at $\boldsymbol{q}=$ $\pm(2 \pi / 3,2 \pi / 3)$, reminiscent of the $120^{\circ}$ spin alignment, as shown in Fig. 2(c). We find in Fig. 2(f) that the peak scales as $S(\boldsymbol{Q}) \propto N_{\mathrm{s}}^{1 / 2}$ in all the regions of the 
spin-liquid phase $\left(0.10<J_{2} / J_{1}<0.135\right)$, implying the emergence of a critical phase without the long-ranged order. In fact, this scaling leads to the spin correlation $\left\langle\boldsymbol{S}_{i} \cdot \boldsymbol{S}_{j}\right\rangle \propto 1 /\left|\boldsymbol{R}_{i}-\boldsymbol{R}_{j}\right|^{20)}$.

At the transition point $J_{2} / J_{1}=0.10(1)$, the spin liquid undergoes a continuous quantum phase transition to the $120^{\circ}$ Néel state, while at $J_{2} / J_{1}=0.135(5)$ a level crossing to the stripe state occurs.

To get further insight into the nature of the spin-liquid ground state, we study the energies of the momentumresolved lowest triplet and singlet excited states. We take the total momenta along the symmetric $\Gamma-\mathrm{K}-\mathrm{M}$ line, namely, $\boldsymbol{K}=(q, q)$ with $0 \leq q \leq \pi$, as illustrated in Fig. 1(d). The ground states have turned out to be always the singlet and stay at the $\Gamma$ point irrespective of $J_{2}$ and $N_{\mathrm{s}}$.

According to the results of spin wave (SW) analyses, ${ }^{21,22)}$ the spin gap $\Delta$ for antiferromagnetic states should scale as $\Delta\left(N_{\mathrm{s}}\right) \propto 1 / N_{\mathrm{s}}$. However, when both the magnetic order and spin gap become zero, especially on continuous transition points, a more subtle scaling dominates. For example, for the two-dimensional quantum Heisenberg model, when a quantum phase transition occurs between the phase with an $\mathrm{O}(3)$ long-ranged order and the disordered phase with a finite-gap spin excitation, the nature of the transition was proposed to be essentially the same as that of the two-dimensional quantum $\mathrm{O}(3)$ nonlinear sigma model. ${ }^{23)}$ Since the correlation length is the same in the spatial and time directions, the dynamical exponent $z$ becomes $z=1$ at the critical point. Therefore, the spin gap, which scales as $\Delta \sim k^{z}$ at the critical point, ${ }^{24)}$ obeys the relation $\Delta \sim k \propto 1 / L$ with $L \equiv N_{\mathrm{s}}^{1 / 2}$. Later on, a possible deconfined criticality was proposed in that case. ${ }^{25,26)}$

In general, the critical state may be characterized by the power-law decay $\Delta \propto 1 / L^{z}$ with the dynamical exponent $z$. As shown in Fig. 3, the spin gap at the critical point $\left(J_{2} / J_{1}=0.1\right)$ consistently shows power-law decay for larger sizes, although it is difficult to estimate the critical exponent of the decay $z$ quantitatively. In finite-size systems, higher-order corrections in the extrapolation of the spin gap are nonnegligible, which makes the extrapolation difficult. Indeed, in a previous study on the triangular lattice system, the spin gap of the spin liquid scales as a concave function of $1 / N_{\mathrm{s}}$ if the system size is not sufficiently large ${ }^{27)}$ although the chiral perturbation theory for the square lattice with the well-established antiferromagnetic order predicts the scaling by $1 / N_{\mathrm{s}}$ with the negative coefficient of the $1 / N_{\mathrm{s}}^{3 / 2}$ correction. ${ }^{21)}$ Therefore, the estimated critical exponents may become larger than the exact exponents.

This critical point turns out to be unusual, because the spin gap appears to decay as a power law even deep inside the spin-liquid phase. Within the available system size $(\leq 18 \times 18)$, these results support the nation that the whole spin-liquid phase may belong to the critical phase. This is consistent with the above critical behavior of the spin correlation, because we expect $\left\langle\boldsymbol{S}_{i} \cdot \boldsymbol{S}_{j}\right\rangle \propto$ $1 /\left|\boldsymbol{R}_{i}-\boldsymbol{R}_{j}\right|^{d+z+\eta-2}$ with $d=2$ and $z+\eta=1$ in the whole spin-liquid phase, as we will detail later.
We now study the momentum-resolved spectra of the excitations $\Delta(\boldsymbol{K})$. Generally, if the ground state has a magnetic long-ranged order, corresponding to the magnon modes, it shows gapless excitations at the $\Gamma$ point as well as at the wave vector $\boldsymbol{Q}$ of $S(\boldsymbol{Q})$ peaks. We have indeed confirmed that the present $\mathrm{mVMC}$ calculation reproduces this expectation.

In the spin-liquid phase, we examine the size dependence of singlet and triplet gaps at $\boldsymbol{K}=(n \pi / 3, n \pi / 3)$ $(n \in \mathbb{Z})$, as shown in Fig. 4(a), which can be calculated for all the system sizes that we studied ${ }^{28}$ ) (see also Supplementary Materials for data at other $k$ points). As shown in Figs. 4(b) and 4(c), we find that both singlet and triplet gaps at all the available momenta systematically and substantially decrease with increasing system size, and natural extrapolations suggest vanishingly and unusually small values if it is nonzero. Even at $\boldsymbol{K}=(\pi / 3, \pi / 3)$, where the gap is largest, a simple linear extrapolation suggests the gap $<0.2 J_{1}$. The gaps at other momenta are likely to be less than $0.1 J_{1}$. To our knowledge, such small gaps in the extended points of the Brillouin zone are not known. In the antiferromagnetic phase, the spin wave excitation usually has dispersions of the order of the exchange coupling $J_{1}$.

Now, we discuss comparisons with previously proposed spin-liquid states. In the present result, the singlet and triplet excitation energies look vanishingly small at all the studied total momenta, including the $\Gamma, \mathrm{K}$, and $\mathrm{M}$ points, as well as the middle of the $\Gamma$ and $K$ points, as shown in Fig. 4(a). If the excitation is gapless at the momenta in a finite area of the Brillouin zone, it can be accounted for by the presence of a large spinon Fermi surface. ${ }^{29,30)}$ Although the present model does not contain itinerant electrons, if the finite-size spin gap scales as $1 / L$, the present spin-liquid state is indeed similar to the expected gap scaling of the lowest particle-hole (Stoner) excitation in the (spinon) Fermi liquid. However, the exponent of the power-law decay of the spin correlation $\left(\sim 1 / r^{\alpha}\right.$ with $\left.\alpha=1\right)$ contradicts the expected behavior in the presence of the Fermi surface, namely, $\left\langle\boldsymbol{S}_{i} \cdot \boldsymbol{S}_{j}\right\rangle \propto 1 /\left|\boldsymbol{R}_{i}-\boldsymbol{R}_{j}\right|^{3} \cdot{ }^{31)}$

When the gap closes only at $\boldsymbol{K}=(n \pi / 3, n \pi / 3)(n \in$ $\mathbb{Z}$ ) or at one of these $\boldsymbol{K}$ points, it is consistent with the algebraic spin liquid. For example, for the Dirac spin liquid whose dispersion scales as $\Delta \propto k^{z}$ with $z=1$, it was discussed that the spin correlation may decay as $1 / r^{1+\eta}$ typically with a nonzero $\eta$ because of the coupling of Dirac spinons to a noncompact U(1) gauge field (see Refs. 32 and 33 and references therein). The coupling depends on the flux pattern of the slave-boson mean-field states, and thus the exponent $\eta$ varies according to lattice model; for instance, it was proposed that $\eta \sim 0.5$ for the projected $d$-wave BCS state ${ }^{33)}$ and $\eta \sim 3$ for the $\mathrm{U}(1)$ Dirac spin liquid on the kagome lattice. ${ }^{34)}$ More generally, it can be conjectured that the algebraic spin liquid is characterized by the $1 / L^{z}$ scaling of the gap closing and the $1 / r^{z+\eta}$ decay of the spin correlation. If $z+\eta=1$, it is consistent with the present result. The vanishingly small excitation energies at extended momentum points naturally lead to the power-law decay of the spin correlation in real space at the period of the corresponding momen- 

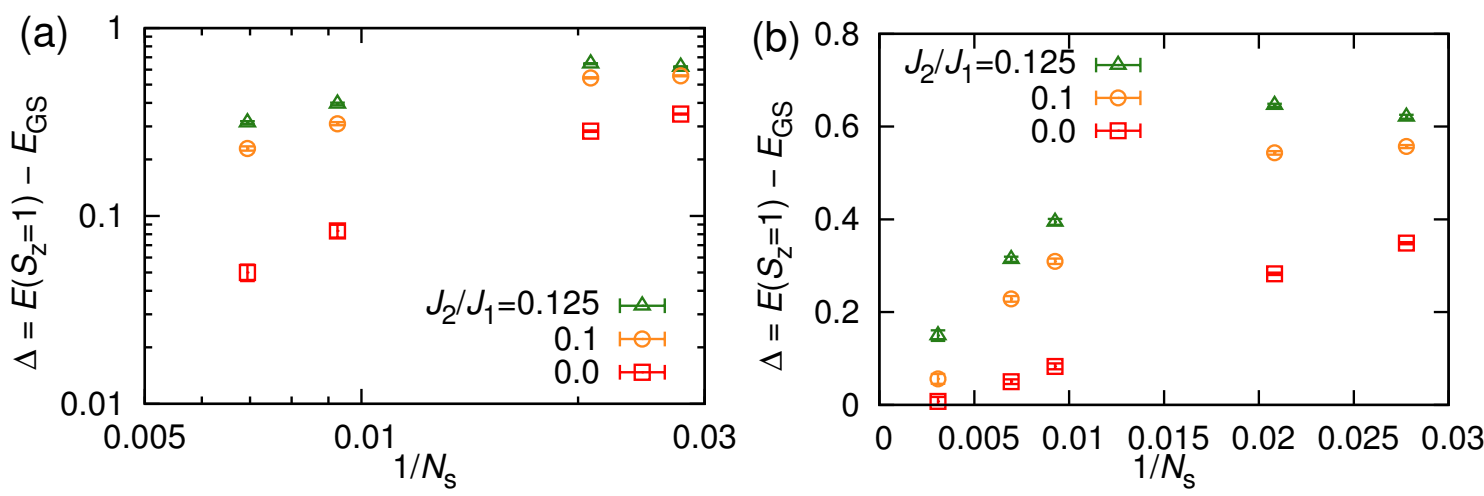

Fig. 3. (color online) Size scalings of spin gaps of $120^{\circ}$ Néel state, spin liquid, and near the critical point. The spin gaps at the $\Gamma$ point are plotted on a log-log plot (a) and a linear plot (b).
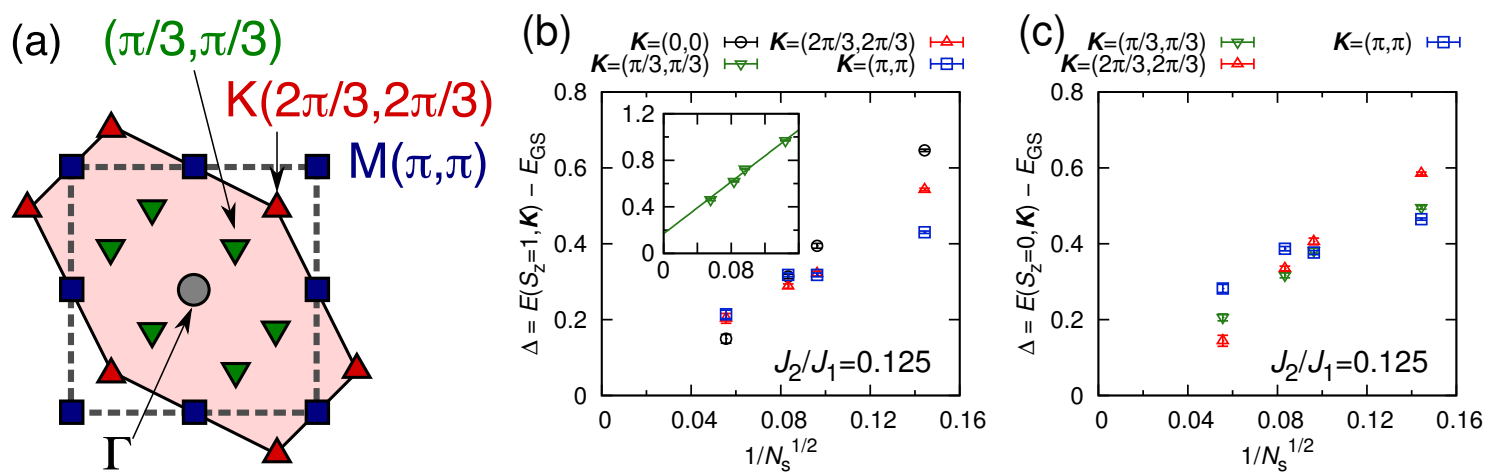

Fig. 4. (color online) Momentum-resolved excitation spectra in spin-liquid phase. (a) Momenta studied for excitation spectra in colors the same as in (b) and (c). Size dependences of triplet (b) and singlet (c) gaps of each total momentum for spin-liquid phase $\left(J_{2} / J_{1}=0.125\right)$. Triplet excitations are gapless only at the $\Gamma$ and $\mathrm{K}$ points for the $120^{\circ}$ Néel state, while they are gapless only at the $\Gamma$ and $\mathrm{M}$ points for the stripe state. For the spin liquid, in addition to the $\mathrm{K}, \mathrm{M}$, and $\Gamma$ points, the middle points of the $\Gamma$ and $\mathrm{K}$ points show unusually small excitation energies.

tum points. However, so far, the clear algebraic $(1 / r)$ decay of the spin correlation is found only for that at the period $(2 \pi / 3,2 \pi / 3)$ ( $120^{\circ}$ order point). This rules out the possibility of a $d$-wave-type spin liquid, ${ }^{16,35)}$ whose gap is open at the period $(2 \pi / 3,2 \pi / 3)$. In the present calculation, the spin correlations of the period at other momentum points decay at least faster than $1 / r^{2}$ because $S(\boldsymbol{q})$ is at most logarithmically divergent and is likely to stay finite in the thermodynamic limit. If this picture applies so that some of the spin correlations at the periods other than $(2 \pi / 3,2 \pi / 3)$ decay exponentially, we expect the opening of gaps at the wavenumber away from the crossing points. So far, the examination of this prediction is not numerically easy with the present computer power. This is left for future studies. Within the available results, the present spin liquid does not contradict the possible algebraic spin liquid or flux state, while our results impose constraints on its nature.

We have shown that the spin- $1 / 2$ Heisenberg model on the triangular lattice with the nearest and nextnearest exchange interactions shows an unusual spin liquid, where the spin correlation decays as $1 / r$ and the finite-size gap closes as a power law as if the quantum critical point is extended to a critical phase. Unusually small gaps at all the total momenta studied here, how- ever, impose a severe constraint on the nature of the spin liquid found in the present study. Clearly, further studies are needed for a more unified understanding of the spin-liquid states found in the present study.

\section{Acknowledgements}

The mVMC codes used for the present computation were based on those first developed by Daisuke Tahara and M. I. and those extended by S. M. and Moyuru Kurita. To compute the Pfaffian of skew-symmetric matrices, we employed PFAPACK. ${ }^{36)}$ The authors thank Takahiro Misawa for fruitful discussions. This work was financially supported by MEXT HPCI Strategic Programs for Innovative Research (SPIRE) and Computational Materials Science Initiative (CMSI). The numerical calculation was partly carried out at the Supercomputer Center, Institute for Solid State Physics, University of Tokyo. The numerical calculation was also partly carried out at $\mathrm{K}$ computer at RIKEN Advanced Institute for Computational Science (AICS) under grant numbers hp120043, hp120283, and hp130007. This work was also supported by a Grant-in-Aid for Scientific Research (Nos. 22104010 and 22340090) from MEXT, Japan. 
1) P. Anderson, Mater. Res. Bull. 8, 153 (1973).

2) B. Bernu, P. Lecheminant, C. Lhuillier, and L. Pierre, Phys. Rev. B 50, 10048 (1994)

3) L. Capriotti, A. E. Trumper, and S. Sorella, Phys. Rev. Lett. 82, 3899 (1999)

4) S. R. White and A. L. Chernyshev, Phys. Rev. Lett. 99, 127004 (2007).

5) Y. Shimizu, K. Miyagawa, K. Kanoda, M. Maesato, and G. Saito, Phys. Rev. Lett. 91, 107001 (2003).

6) T. Itou, A. Oyamada, S. Maegawa, M. Tamura, and R. Kato, Phys. Rev. B 77, 104413 (2008).

7) G. Baskaran, Phys. Rev. Lett. 63, 2524 (1989).

8) N. B. Ivanov, Phys. Rev. B 47, 9105 (1993).

9) S. E. Korshunov, Phys. Rev. B 47, 6165 (1993).

10) L. O. Manuel and H. A. Ceccatto, Phys. Rev. B 60, 9489 (1999).

11) T. Jolicoeur, E. Dagotto, E. Gagliano, and S. Bacci, Phys. Rev. B 42, 4800 (1990).

12) A. V. Chubukov and T. Jolicoeur, Phys. Rev. B 46, 11137 (1992).

13) R. Deutscher and H. Everts, Z. Phys. B 93, 77 (1993).

14) P. Lecheminant, B. Bernu, C. Lhuillier, and L. Pierre, Phys. Rev. B 52, 6647 (1995).

15) P. Sindzingre, B. Bernu, and P. Lecheminant, J. Phys.: Cond. Mat. 7, 8805 (1995).

16) R. V. Mishmash, J. R. Garrison, S. Bieri, and C. Xu, Phys. Rev. Lett. 111, 157203 (2013)

17) D. Tahara and M. Imada, J. Phys. Soc. Jpn. 77, 114701 (2008).

18) (Supplemental Material) Details of the choice of the wave functions are described in Supplemental Materials.

19) D. Heidarian, S. Sorella, and F. Becca, Phys. Rev. B 80 012404 (2009)

20) (Supplemental Material) The power-law decay of spin correlation function in the spin liquid phase is discussed in Supplemental Materials.

21) P. Hasenfratz and F. Niedermayer, Z. Phys. B 92, 91 (1993).

22) H. Neuberger and T. Ziman, Phys. Rev. B 39, 2608 (1989).

23) M. Troyer, M. Imada, and K. Ueda, J. Phys. Soc. Jpn. 66, 2957 (1997)

24) A. W. Sandvik, AIP Conf. Proc. 1297, 135 (2010).

25) T. Senthil, A. Vishwanath, L. Balents, S. Sachdev, and M. P. A. Fisher, Science 303, 1490 (2004).

26) T. Senthil, L. Balents, S. Sachdev, A. Vishwanath, and M. P. A. Fisher, Phys. Rev. B 70, 144407 (2004).

27) T. Mizusaki and M. Imada, Phys. Rev. B 74, 014421 (2006).

28) (Supplemental Material) The gaps other than points at $\boldsymbol{k}=$ $(n \pi / 3, n \pi / 3)(n \in \mathbb{Z})$ for the finite-size systems are also discussed in Supplemental Materials.

29) O. I. Motrunich, Phys. Rev. B 72, 045105 (2005).

30) S.-S. Lee and P. A. Lee, Phys. Rev. Lett. 95, 036403 (2005).

31) N. Furukawa and M. Imada, J. Phys. Soc. Jpn. 61, 3331 (1992)

32) W. Rantner and X.-G. Wen, Phys. Rev. Lett. 86, 3871 (2001).

33) M. Hermele, T. Senthil, and M. P. A. Fisher, Phys. Rev. B 72, 104404 (2005).

34) M. Hermele, Y. Ran, P. A. Lee, and X.-G. Wen, Phys. Rev. B 77, 224413 (2008)

35) T. Grover, N. Trivedi, T. Senthil, and P. A. Lee, Phys. Rev B 81, 245121 (2010).

36) M. Wimmer, ACM Trans. Math. Softw. 38, 30 (2012). 


\section{Supplemental Materials to "Gapless spin-liquid phase in an extended spin $1 / 2$ triangular Heisenberg model"}

\section{Variational Monte Carlo method}

We consider the variational wave function in the form $|\Psi\rangle=\mathcal{P} \mathcal{L}\left|\Psi_{0}\right\rangle$ with

$$
\left|\Psi_{0}\right\rangle \equiv\left(\sum_{i, j=1}^{N_{\mathrm{s}}} \sum_{s, t=\uparrow \text { or } \downarrow} f_{i j}^{s t} c_{i s}^{\dagger} c_{j t}^{\dagger}\right)^{N_{\mathrm{e}} / 2}|0\rangle,
$$

which can describe antiferromagnetic states with collinear, noncollinear, and even noncoplanar spin alignment, and also gapless and gapped spin-liquid states on an equal footing. Here, $\left|\Psi_{0}\right\rangle$ is a generalized Bardeen-Cooper-Schrieffer (BCS) wave function, where $f_{i j}^{s t}$ and $|0\rangle$ denote the variational parameter and the vacuum. This is a natural extension of the Liang-Doucot-Anderson type wave function ${ }^{1)}$ and the Hartree-FockBogoliubov type wave function. ${ }^{2,3)}$ Note that not only the product of the paired singlet but also the paired triplet component are allowed in this scheme beyond the RVB singlet, ${ }^{1)}$ which is crucial in representing noncollinear spin order. We assume $f_{i j}^{s t}$ to be long-ranged and allow symmetry breaking, namely six-sublattice structure. To restore the translational symmetry of $\left|\Psi_{0}\right\rangle$, we consider the total momentum projection

$$
\mathcal{L}^{\boldsymbol{K}}=\frac{1}{N_{\mathrm{s}}} \sum_{\boldsymbol{R}} e^{i \boldsymbol{K} \cdot \boldsymbol{R}} T_{\boldsymbol{R}}
$$

where $\boldsymbol{K}$ and $T_{\boldsymbol{R}}$ denote the total momentum [defined in Fig. 1(d)] and the translation operator, respectively. We apply the projection onto all the possible total momenta. In addition to the Gutzwiller factor

$$
\mathcal{P}_{\mathrm{G}}=\prod_{i}\left(1-n_{i \uparrow} n_{i \downarrow}\right)
$$

with $n_{i s}=c_{i s}^{\dagger} c_{i s}$, which excludes the double occupation of the electron, to reach a wave function closer to the true ground state (lowest triplet state), we also impose a projection $\mathcal{P}_{S_{\text {tot }}^{z}=0}\left(\mathcal{P}_{S_{\text {tot }}^{z}=1}\right)$ on the wave function by keeping the difference in the number of up and down spins fixed as zero (two). We consider lattices for $N_{\mathrm{s}}=L \times L$ and $\sqrt{3} L \times \sqrt{3} L$ up to $18 \times 18$ sites under the periodic boundary condition. By using the stochastic reconfiguration (SR) method, ${ }^{4}$ we optimize the parameters by typically 2000 SR steps. After confirming the energy convergence, we calculate the expectation values of the physical quantities and average them over more than 10 independent runs containing typically 2000 Monte Carlo steps each to estimate statistical errors. 


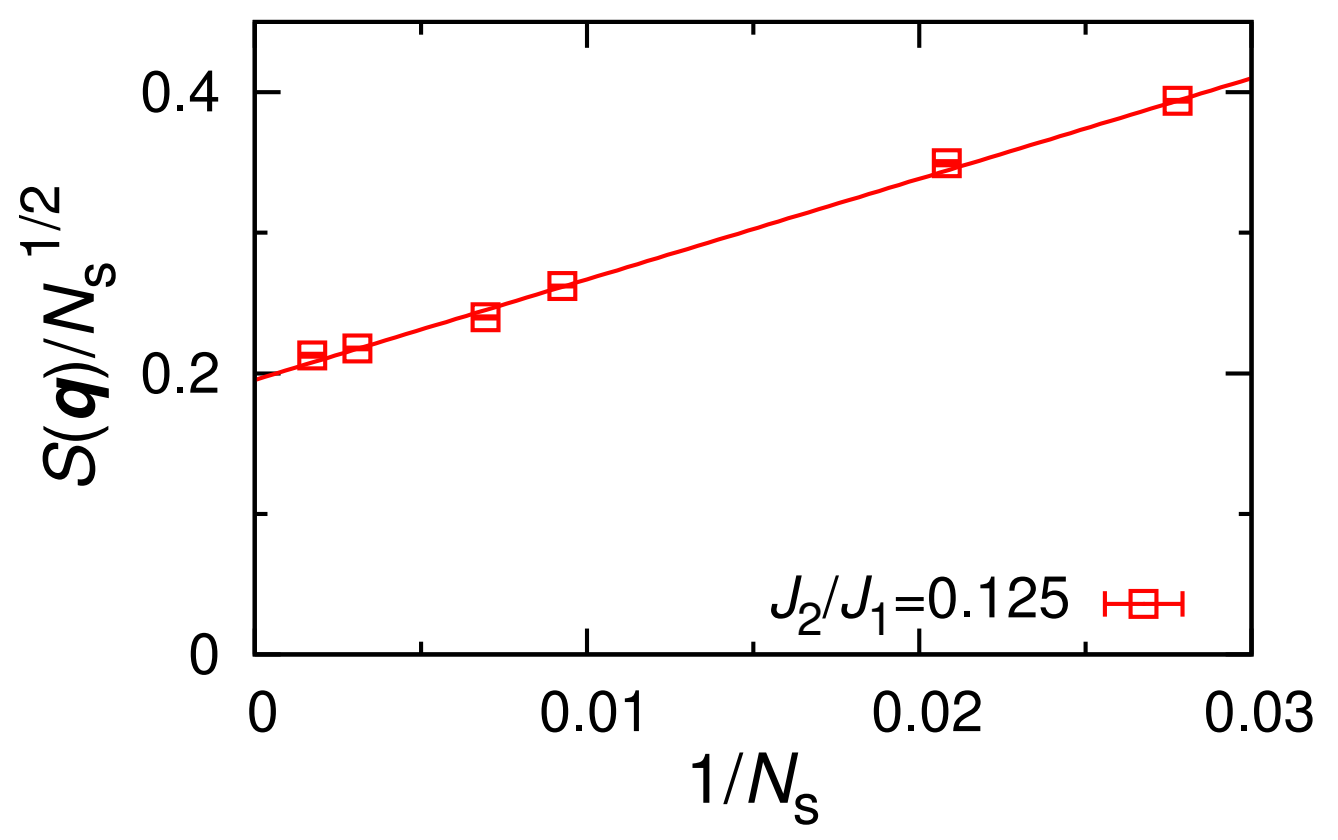

Fig. S.1. (color online) Size dependence of peak value of spin structure factor divided by square root of system size. The data are calculated up to $24 \times 24$ sites at $J_{2} / J_{1}=0.125$. It is well fit by the scaling $S(\boldsymbol{q}) / N_{\mathrm{s}}^{1 / 2}=S_{1}+S_{2} / N_{\mathrm{s}}$, where $S_{1}$ and $S_{2}$ are constants.

\section{Spin correlation function in spin-liquid phase}

Here, we discuss the spin correlation function in the spin-liquid phase. In the Ginzburg-Landau theory, the spin correlation function at the critical point is given as $C(r) \propto r^{-(d-2)}$, where $d(>2)$ is the spatial dimension. Beyond the Ginzburg-Landau theory, at the critical point and inside the critical phase, the correction may be given by the dynamical exponent $z$ and the anomalous dimension $\eta$ as $C(r) \propto r^{-(d+z+\eta-2)}$ $(d+z+\eta>2) .^{5,6)}$ This also leads to $S\left(Q, N_{\mathrm{s}}\right) \propto N_{\mathrm{s}}^{2-(d+z+\eta) / 2}$ for $d+z+\eta<4$. Figure S.1 shows that the spin liquid follows the critical scaling with $z+\eta \sim 1$.

\section{Excitation energies at $k$-points other than $k=(n \pi / 3, n \pi / 3)$}

To understand the possible form of the excitation energy, we also investigate the excitation energies for $N_{\mathrm{s}}=6 \sqrt{3} \times 6 \sqrt{3}$ and $12 \times 12$ at the $\boldsymbol{k}=(q, q)$ with $q$ being achievable wave vectors. As shown in Fig. S.2, along the $\boldsymbol{k}=(q, q)$ line for the finite-size systems, the singlet excitations appear to have a peak around $\boldsymbol{k}=(\pi / 2, \pi / 2)$. On the other hand, the triplet excitations appear to have a broad beak around $\boldsymbol{k}=(\pi / 3, \pi / 3)$. The size of these excitation energies are less than or nearly comparable to the larger triplet gap at $\boldsymbol{k}=(\pi / 3, \pi / 3)$. Although it is difficult to estimate the dispersion in the 

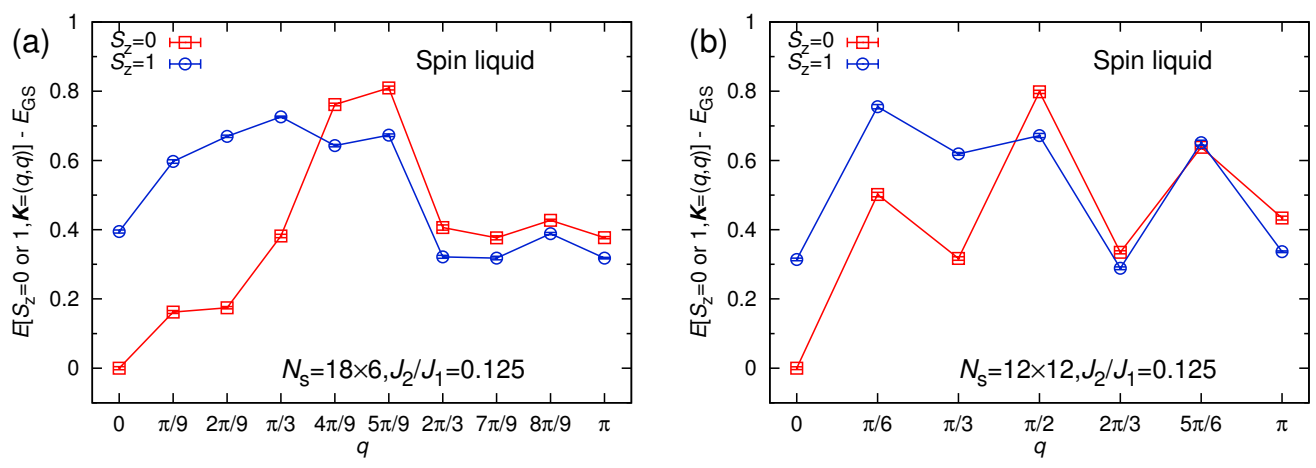

Fig. S. 2. (color online) Total momentum dependence of singlet and triplet energies of spin liquid for system sizes (a) $N_{\mathrm{s}}=6 \sqrt{3} \times 6 \sqrt{3}$ and (b) $N_{\mathrm{s}}=12 \times 12$. The red squares and the blue circles denote the singlet and triplet energies with the total momentum $\boldsymbol{K}=(q, q)$. The lines are guide for the eye.

thermodynamic limit, since even the larger triplet gap at $\boldsymbol{k}=(\pi / 3, \pi / 3)$ converges to a tiny value $\left(\Delta<0.2 J_{1}\right)$, it is likely that almost all of the excitations along $\boldsymbol{k}=(q, q)$ line converges to a near zero value. In this case, it can be conjectured that nearly dispersion-less excitations appear along the $\Gamma-\mathrm{K}$ lines and the $\mathrm{K}-\mathrm{M}$ lines. 


\section{References}

1) S. Liang, B. Doucot, and P. W. Anderson, Phys. Rev. Lett. 61, 365 (1988).

2) T. Giamarchi and C. Lhuillier, Phys. Rev. B 43, 12943 (1991).

3) A. Himeda and M. Ogata, Phys. Rev. Lett. 85, 4345 (2000).

4) S. Sorella, Phys. Rev. Lett. 80, 4558 (1998).

5) N. Goldenfeld, Lectures on Phase Transitions and the Renormalization Group (Westview Press, Boulder, CO, 1992).

6) J. Cardy, Scaling and Renormalization in Statistical Physics (Cambridge University Press, Cambridge, 1996). 